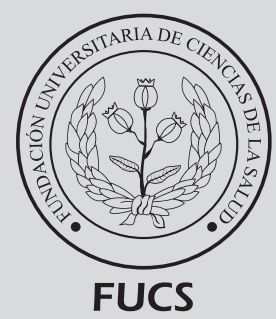

\title{
Estimación de las tasas de mortalidad y letalidad por COVID-19 en Colombia
}

\section{Estimating mortality and lethality rate from COVID-19 in Colombia}

Ingeniero. Magister en Gestión de la Tecnología Educativa, Especialista en Administración de la Informática Educativa.

Docente de matemáticas e Investigador, Secretaría de Educación de Soacha, Cundinamarca.

\section{R E S U M EN}

Introducción: el mundo está en aguas inéditas para la temporada 2020 del virus respiratorio. Por primera vez en la historia moderna, el mundo afronta la posibilidad de la pandemia de la enfermedad por coronavirus 2019 (COVID-19) y una epidemia simultánea de influenza estacional. Objetivo: presentar un método para calcular las tasas de mortalidad y letalidad en los diferentes departamentos de Colombia por COVID-19. Metodología: para desarrollar la investigación se utilizó la base de datos de las personas fallecidas por COVID-19; la información de los datos corresponde a la base de acumulada hasta el 10 de agosto de 2020. Resultados: el grupo etario que presentó mayor tasa de mortalidad es la de 90-99, con 338 muertes por cada 100.000 habitantes, seguido del grupo entre 80-89, con 335 muertes por cada 100 habitantes, y los grupos con menor tasa de mortalidad de 0-9 y 10-19, que no alcanzan a tener una sola muerte por cada 100.000 habitantes. A nivel nacional la tasa por cada 100.000 habitantes fue 26 muertes. Conclusiones: el rastreo al exceso de mortalidad y la exploración activa de casos patentados con diagnósticos confirmados y presuntivos de infección por COVID-19, son necesarios para comprobar cuales funciones suceden por causa directa del coronavirus COVID-19, con una infección concomitante, o por causas diferentes.

Palabras clave: coronavirus, mortalidad, letalidad, COVID-19.

(C) 2020 Fundación Universitaria de Ciencias de la Salud - FUCS. Este es un artículo Open Access bajo la licencia CC BY-NC-ND (http://creativecommons.org/licenses/by-nc-nd/4.0/).

\section{INFORMACIÓN DEL ARTÍCULO}

Historia del artículo:

Fecha recibido: agosto 18 de 2020 Fecha aceptado: agosto 21 de 2020

\footnotetext{
Autor para correspondencia.

Jorge Enrique Díaz Pinzón jediazp@unal.edu.co
}

DOI

10.31260/RepertMedCir.01217372.1103 


\section{A BSTRACT}

Introduction: the world is in an unprecedented scenario for the 2020 season of respiratory viruses. For the first time in modern history, the world is facing the possibility of a coronavirus disease (COVID-19) pandemic and a simultaneous epidemic of seasonal influenza. Objective: to present a method for calculating the mortality and lethality rates attributed to COVID-19 infection in the different departments of Colombia. Methodology: The database including people who have died from COVID-19 infection was used for the research, with the information accumulated up to August 10 2020. Results: the age group with the highest mortality rate is the 90-99 years group with 338 deaths per 100.000 inhabitants, followed by the 80-89 years group, with 335 deaths per 100.000 inhabitants. The groups with the lowest mortality rate are the 0-9 and 10-19 age groups, with less than 1 death per 100.000 inhabitants. Nationally, the rate per 100.000 inhabitants was 26 deaths. Conclusions: there is a need of tracking excess mortality data and performing an active exploration of patented cases with confirmed or presumptive diagnosis of COVID-19 infection, to check which deaths were caused by the COVID-19 coronavirus itself, a concomitant infection, or by other causes.

Key words: coronavirus, mortality, lethality, COVID-19.

(C) 2020 Fundación Universitaria de Ciencias de la Salud - FUCS. This is an open access article under the CC BY-NC-ND license (http://creativecommons.org/licenses/by-nc-nd/4.0/).

\section{INTRODUCCIÓN}

El mundo está en aguas inéditas para la temporada 2020 del virus respiratorio. Por primera vez en la historia moderna, el mundo afronta la posibilidad de la pandemia de la enfermedad por coronavirus 2019 (COVID-19) y una epidemia simultánea de influenza estacional. Cada uno causa enfermedades y muertes que intimidan la vida, especialmente en adultos mayores, personas con enfermedades crónicas y otras poblaciones vulnerables. ${ }^{1,2}$

Los pacientes contagiados presentan una extensa gama de síntomas, desde neumonía asintomática por completo hasta neumonía progresiva que conduce a la muerte. Las pruebas rigurosas y divulgadas siguen siendo un componente crítico de las estrategias para contener esta pandemia. La disponibilidad restringida de diagnósticos moleculares limita el uso de estas tecnologías a quienes se muestran con enfermedades. ${ }^{3}$ Ahora, el COVID-19, causado por el nuevo coronavirus 2 del síndrome respiratorio agudo severo (SARS-CoV-2), es una pandemia incesante que ha tensado y en algunos lugares, agobiado los sistemas de salud. ${ }^{4}$ A medida que la enfermedad por coronavirus 2019 (COVID-19) se ha desarrollado, la salud pública y el bienestar económico están cada vez más en aprieto. Los gobiernos están priorizando la salud pública, pero el procedimiento actual y el aislamiento social, es costoso ya que el comercio sigue cerrado. ${ }^{5}$ Esta pandemia mundial ha acentuado la necesidad de comprender cómo es que sobrevivimos a las infecciones y por qué esto puede ser dispar de la forma en que a menudo pensamos sobre el tratamiento de enfermedades infecciosas ${ }^{6}$. La OMS tiene un enfoque similar en la caracterización de terapias que destruyen el virus con el lanzamiento del ensayo SOLIDARITY que está facilitando la prueba de múltiples estrategias antivirales que pueden ser seguras contra el COVID-19. ${ }^{7}$ Para cualquier brote dado, no precisamente conocemos el patógeno de antemano y por lo tanto, es factible que no cuente con vacunas y antimicrobianos efectivos. Sin embargo, sabemos cómo actúa en el cuerpo y que a pesar de la causa primordial de la enfermedad, hay un número finito de formas en que un paciente puede desarrollar esta patología y morir. ${ }^{8}$ De acuerdo con el estudio de investigación realizado por Díaz ${ }^{9}$, el mayor número de personas fallecidas a 30 de junio 2020, lo lideró Estados Unidos con 128.581, Brasil 58.385, Rusia 47.730, Italia 34.767, Francia 29.843, España 28.355, y México 27.121.

La solución a todo esto no está clara. Los científicos que trabajan en regímenes restrictivos obtienen nuestro apoyo y admiración, y la colaboración científica con científicos chinos ha sido decisivo para comprender el brote y la biología del virus. Prevenir una pandemia es improbable sin la colaboración internacional. ${ }^{10}$

\section{MORTALIDAD GENERAL}

La mortalidad general es el volumen de muertes ocurridas por todas las causas de la enfermedad, en todos los grupos de edad y para ambos sexos. La mortalidad general, que comúnmente se expresa en forma de tasa, puede ser cruda o ajustada, de acuerdo con el tratamiento estadístico que reciba. La tasa cruda de mortalidad se calcula de la siguiente manera: ${ }^{11,12}$ :

Número de muertes

Tasa de mortalidad específica $=\frac{\text { en un periodo determinado }}{\text { Población total promedio en }}(x 1000)$ el mismo periodo 
TASA DE LETALIDAD

La letalidad en sentido exacto es una proporción ya que enuncia el número de defunciones entre el número de casos del cual estas forman parte. Sin embargo, universalmente se expresa como tasa de letalidad y se registra como el porcentaje de muertes de una causa específica con respecto al total de enfermos por esa causa. ${ }^{11,12}$ : la letalidad es una medida de la gravedad de una enfermedad estimada desde el punto de vista poblacional y se precisa como la proporción de casos de una enfermedad que surgen mortales con respecto al total de casos en un periodo determinado. La medida muestra la relevancia de la enfermedad en términos de su disposición para producir la muerte y se deduce de la siguiente manera:

\section{Letalidad $(\%)=\frac{\begin{array}{c}\text { (Número de muertes por una enfermedad } \\ \text { en un periodo determinado) }\end{array}}{\text { (Número de casos diagnosticados de }}(\mathbf{x 1 0 0 0 )}$ la misma enfermedad en el mismo periodo)}

\section{RES ULTA D O S}

En la tabla 1 se determinó el método de cálculo de las tasas de mortalidad por COVID-19 por grupo etario en Colombia, con la información recolectada por parte del Instituto Nacional de Salud ${ }^{13}$, con fecha de corte a 10 de agosto de 2020, además de la información de la población proyectada por grupo etario del $\mathrm{DANE}^{14}$ y el cálculo de las tasas de mortalidad por parte de la Organización Mundial de la Salud ${ }^{15}$ por cada 100.000 habitantes.

Tabla 1. Método de cálculo de las tasas de mortalidad por COVID-19, por grupo etario en Colombia

\begin{tabular}{|c|c|c|c|c|c|}
\hline $\begin{array}{l}\text { Grupo } \\
\text { Etario }\end{array}$ & $\begin{array}{l}\text { Número de } \\
\text { muertes }\end{array}$ & $\begin{array}{c}\text { Población por } \\
\text { grupo etario }\end{array}$ & $\begin{array}{l}\text { Cociente } \\
\text { (1) / (2) }\end{array}$ & Base & $\begin{array}{c}\text { Tasa/100000 } \\
\text { habitantes }(3) \times(4)\end{array}$ \\
\hline Años & (1) & (2) & V & (4) & 3 \\
\hline $0-9$ & 25 & 7'863.825 & $3,17911 \mathrm{E}-06$ & 100000 & 0,31791145 \\
\hline $10-19$ & 17 & 8112.327 & $2,09558 \mathrm{E}-06$ & 100000 & 0,20955763 \\
\hline $20-29$ & 161 & $8^{\prime} 551.856$ & $1,88263 \mathrm{E}-05$ & 100000 & 1,88263226 \\
\hline $30-39$ & 412 & $7 ' 470.681$ & $5,51489 \mathrm{E}-05$ & 100000 & 5,51489215 \\
\hline $40-49$ & 925 & $6 ' 130.204$ & 0,000150892 & 100000 & 15,0892205 \\
\hline $50-59$ & 1865 & $5^{\prime} 434.890$ & 0,000343153 & 100000 & 34,3153219 \\
\hline $60-69$ & 3062 & 3'795.322 & 0,000806783 & 100000 & 80,6782666 \\
\hline $70-79$ & 3369 & 2'003.827 & 0,001681283 & 100000 & 168,128287 \\
\hline $80-89$ & 2610 & 777.513 & 0,003356857 & 100000 & 335,685706 \\
\hline $90-99$ & 708 & 209.034 & 0,003387009 & 100000 & 338,700881 \\
\hline Total & 13.154 & $50 ’ 349.479$ & 0,000261254 & & 26,1253945 \\
\hline
\end{tabular}

Fuente: el autor.

Se evidencia que el grupo etario que presenta mayor tasa de mortalidad es el de 90-99 con 338 muertes por cada 100.000 habitantes, seguido del grupo entre 80-89 con 335 muertes por cada 100 habitantes, y los grupos con menor tasa de mortalidad de 0-9 y 10-19, que no alcanzan a tener una sola muerte por cada 100.000 habitantes. A nivel nacional la tasa por cada 100.000 habitantes es de 26 muertes.
En la tabla 2 se determinaron las tasas de mortalidad específicas (TME) por COVID-19, por departamentos en Colombia. Con la información recolectada por parte del Instituto Nacional de Salud 13 se aprecia que el departamento con mayor TME por cada 1000 habitantes es Amazonas con una tasa de 1,31 y Atlántico con 0,99 es decir en estos dos departamentos 1 de cada 1000 habitantes muere por COVID-19. Solo tres departamentos presentan TME igual a cero, San Andrés, Guaviare y Vichada. A nivel nacional la TME equivale al 0,26.

Tabla 2. Tasa de mortalidad por COVID-19 por departamentos. Colombia a 10 de agosto 2020

\begin{tabular}{|c|c|c|c|}
\hline Departamentos & Total de habitantes & Fallecidos & Mortalidad \\
\hline Nacional & 50372424 & 13154 & 0,26113494 \\
\hline Bogotá & 7743955 & 3778 & 0,48786441 \\
\hline Valle del Cauca & 4532152 & 1242 & 0,274042 \\
\hline Atlántico & 2722128 & 2699 & 0,99150371 \\
\hline Amazonas & 79020 & 104 & 1,3161225 \\
\hline Nariño & 1627589 & 345 & 0,21196998 \\
\hline Antioquia & 6677930 & 903 & 0,13522154 \\
\hline Cundinamarca & 3242999 & 327 & 0,10083259 \\
\hline Meta & 1063454 & 57 & 0,05359893 \\
\hline Choco & 544764 & 119 & 0,21844322 \\
\hline Cesar & 1295387 & 100 & 0,07719701 \\
\hline Magdalena & 1427026 & 438 & 0,30693204 \\
\hline Tolima & 1339998 & 84 & 0,06268666 \\
\hline Bolívar & 2180976 & 630 & 0,2888615 \\
\hline Risaralda & 961055 & 51 & 0,05306668 \\
\hline Huila & 1122622 & 42 & 0,03741241 \\
\hline Córdoba & 1828947 & 852 & 0,46584182 \\
\hline Boyacá & 1242731 & 39 & 0,0313825 \\
\hline Santander & 2280908 & 266 & 0,11662022 \\
\hline Sucre & 949252 & 395 & 0,41611711 \\
\hline Caldas & 1018453 & 18 & 0,01767386 \\
\hline Cauca & 1491937 & 96 & 0,06434588 \\
\hline N Santander & 1620318 & 262 & 0,16169665 \\
\hline Quindío & 555401 & 13 & 0,02340651 \\
\hline La Guajira & 965718 & 146 & 0,15118285 \\
\hline Casanare & 435195 & 7 & 0,01608474 \\
\hline Vaupés & 44712 & 1 & 0,02236536 \\
\hline Caquetá & 410521 & 68 & 0,16564317 \\
\hline San Andrés & 63692 & 0 & 0 \\
\hline Putumayo & 359127 & 63 & 0,17542541 \\
\hline Guainía & 50636 & 1 & 0,0197488 \\
\hline Guaviare & 86657 & 0 & 0 \\
\hline Arauca & 294206 & 8 & 0,02719183 \\
\hline Vichada & 112958 & 0 & 0 \\
\hline
\end{tabular}

Fuente: el autor.

En la tabla 3 se establecieron las tasas de letalidad por COVID-19, por departamentos en Colombia. Con la información recolectada por parte del Instituto Nacional de Salud $^{13}$ se aprecia que aquellos con mayor tasa de letalidad por cada 100 habitantes está Córdoba en primer lugar con una tasa de 8,22 es decir que 8 de cada 100 casos positivos fallece, le sigue Guainía con un 7,14 es decir que 7 de cada 100 casos positivos fallece, continuando Norte de Santander con 5,38; La Guajira con 5,23; Sucre 5,21 y 
Magdalena con 5,12. En estos tres últimos departamentos 5 de cada 100 casos positivos por COVID-19 fallecen. Solo tres departamentos presentan tasa de letalidad igual a cero San Andrés, Guaviare y Vichada. A nivel nacional se observa una tasa de letalidad de 3,30, es decir que 3 de cada 100 casos positivos fallece.

Tabla 3. Tasa de letalidad por COVID-19 por departamentos. Colombia a 10 de agosto 2020

\begin{tabular}{|c|c|c|c|}
\hline Departamentos & Casos activos & Fallecidos & Letalidad \\
\hline Nacional & 397623 & 13154 & 3,30 \\
\hline Bogotá & 136764 & 3778 & 2,76 \\
\hline Valle del Cauca & 31951 & 1242 & 3,88 \\
\hline Atlántico & 58634 & 2699 & 4,60 \\
\hline Amazonas & 2615 & 104 & 3,97 \\
\hline Nariño & 9898 & 345 & 3,48 \\
\hline Antioquia & 52417 & 903 & 1,72 \\
\hline Cundinamarca & 12092 & 327 & 2,70 \\
\hline Meta & 4111 & 57 & 1,38 \\
\hline Choco & 3265 & 119 & 3,64 \\
\hline Cesar & 4511 & 100 & 2,21 \\
\hline Magdalena & 8547 & 438 & 5,12 \\
\hline Tolima & 3681 & 84 & 2,28 \\
\hline Bolivar & 20456 & 630 & 3,07 \\
\hline Risaralda & 2704 & 51 & 1,88 \\
\hline Huila & 1747 & 42 & 2,40 \\
\hline Córdoba & 10361 & 852 & 8,22 \\
\hline Boyacá & 1621 & 39 & 2,40 \\
\hline Santander & 6958 & 266 & 3,82 \\
\hline Sucre & 7575 & 395 & 5,21 \\
\hline Caldas & 1383 & 18 & 1,30 \\
\hline Cauca & 2681 & 96 & 3,58 \\
\hline N Santander & 4868 & 262 & 5,38 \\
\hline Quindío & 528 & 13 & 2,46 \\
\hline La Guajira & 2787 & 146 & 5,23 \\
\hline Casanare & 491 & 7 & 1,42 \\
\hline Vaupés & 61 & 1 & 1,63 \\
\hline Caquetá & 2619 & 68 & 2,59 \\
\hline San Andrés & 51 & 0 & 0 \\
\hline Putumayo & 1572 & 63 & 4,00 \\
\hline Guainía & 14 & 1 & 7,14 \\
\hline Guaviare & 160 & 0 & 0 \\
\hline Arauca & 495 & 8 & 1,61 \\
\hline Vichada & 5 & 0 & 0 \\
\hline
\end{tabular}

Fuente: el autor.

\section{CONCLUSIONES}

Se evidenció que al 10 de agosto de 2020, el grupo etario que presentó mayor tasa de mortalidad es el de 90-99 con 338 muertes por cada 100.000 habitantes, seguido del grupo entre 80-89 con 335 muertes por cada 100 habitantes y los grupos con menor tasa de mortalidad de 0-9 y 1019 no alcanzan a tener una sola muerte por cada 100.000 habitantes. A nivel nacional la tasa por cada 100.000 habitantes fueron 26 muertes.

Se determinó que el departamento con mayor TME por cada 1000 habitantes fue Amazonas en primer lugar con una tasa de 1,31, y Atlántico con un 0,99 es decir en estos dos departamentos 1 de cada 1000 habitantes muere por COVID-19. Solo tres departamentos presentan TME igual a cero, San Andrés, Guaviare y Vichada. A nivel nacional la TME equivale al 0,26 . Ahora, se evaluó que los departamentos con mayor tasa de letalidad por cada 100 habitantes está en primer lugar Córdoba con una tasa de 8,22 , es decir que 8 de cada 100 casos positivos fallece, le sigue Guainía con un 7,14 es decir que 7 de cada 100 casos positivos fallece, continuando Norte de Santander con 5,38; La Guajira con 5,23; Sucre 5,21 y Magdalena con 5,12. En estos tres últimos departamentos 5 de cada 100 casos positivos por COVID-19 fallecen. Solo tres departamentos presentan tasa de letalidad igual a cero, San Andrés, Guaviare y Vichada. A nivel nacional se observó una tasa de letalidad de 3,30, es decir que 3 de cada 100 casos positivos fallece en Colombia. El rastreo al exceso de mortalidad y la exploración activa de casos patentados con diagnósticos confirmados y presuntivos de infección por COVID-19, son necesarios para comprobar que defunciones suceden por causa directa del coronavirus COVID-19, con una infección concomitante, o por causas diferentes. ${ }^{16}$

\section{REFEREN CIAS}

1. Belongia EA, Osterholm MT. COVID-19 and flu, a perfect storm. Science. 2020;368(6496):1163. doi: 10.1126/science.abd2220

2. Wigginton NS, Cunningham RM, Katz RH, Lidstrom ME, Moler KA, Wirtz D, et al. Moving academic research forward during COVID-19. Science. 2020;368(6496):1190-2. doi: 10.1126/science. abc5599

3. Jeong H, Rogers JA, Xu S. Continuous on-body sensing for the COVID-19 pandemic: Gaps and opportunities. Sci Adv. 2020:1-5. doi: 10.1126/sciadv.abd4794

4. Singer BD. COVID-19 and the next influenza season. Science advances. 2020;6(31):eabd0086. doi: 10.1126/sciadv.abd0086

5. Azoulay P, Jones B. Beat COVID-19 through innovation. Science. 2020;368(6491):553. doi: 10.1126/science. abc5792

6. Ayres JS. Surviving COVID-19: A disease tolerance perspective. Science advances. 2020;6(18):eabc1518. doi: 10.1126/sciadv. abc1518

7. Kupferschmidt K, Cohen J. Race to find COVID-19 treatments accelerates. Science. 2020;367(6485):1412-3. doi: 10.1126/ science.367.6485.1412

8. Ayres JS. The Biology of Physiological Health. Cell. 2020;181(2):25069. doi: 10.1016/j.cell.2020.03.036

9. Díaz Pinzón JE. Estudio de los resultados del contagio por COVID-19 a nivel mundial. Repert Med Cir. 2020;29(Supl. Núm. 1):65-71. doi: 10.31260/ RepertMedCir.01217372.1089

10. Thorp HH. The costs of secrecy. Science. 2020;367(6481):959. doi: $10.1126 /$ science.abb4420 
11. Moreno-Altamirano A, López-Moreno S, Corcho-Berdugo A. Principales medidas en epidemiología. Salud Pública de Méx. 2000;42(4):337-48.

12. Quintana-Salgado L. Medidas de frecuencia en epidemiología [Internet]. 2015 [citado 2020 julio 25]; Disponible en: https://es.slideshare.net/lualberts20/medidas-de-frecuencia-enepidemiologa-2015.

13. Instituto Nacional de Salud. Coronavirus (CO-VID-19) en Colombia [Internet]. Colombia: Instituto Nacional de Salud; 2020 [citado 2020 julio 25]; Disponible en: https://bit. ly/2UNnOtl
14. Departamento Administrativo Nacional de Estadística. Proyecciones y retroproyecciones de población [Internet]. Colombia: Departamento Administrativo Nacional de Estadística DANE; 2000 [citado 2020 julio 25]; Disponible en: https://www. dane.gov.co/index.php/estadisticas-por-tema/demografia-ypoblacion/proyecciones-de-poblacion.

15. Organización Mundial de la Salud, Organización Panamericana de la Salud. Indicadores de salud: Aspectos conceptuales y operativos. Organización Mundial de la Salud; 2020.

16. Alvarez Castaño VH. Exceso de mortalidad en Colombia 2020. Bogotá: Ministerio de Salud y Protección Social; 2020. 